\title{
The Research on Teaching Model of Flipped Classroom Based on Rain Classroom
}

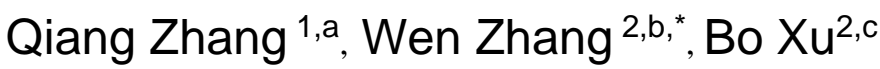

${ }^{1}$ Science and Technology on Underwater Vehicle Laboratory, Harbin Engineering University, Harbin China

${ }^{2}$ College of Automation, Harbin Engineering University, Harbin China

azhangqiang103@hrbeu.edu.cn, bzhangwen@hrbeu.edu.cn, `xubo@hrbeu.edu.cn

${ }^{*}$ Corresponding author

Keywords: rain classroom, flip class, teaching system, teaching program, assessment method.

\begin{abstract}
To slove the problem of the popularization difficulty in flipped classroom, we proposed the teaching model of flipped classrom based on rain classrom, for which we introduced scientific and objective approaches of teaching program design and assessment method. The teaching experiment result indicates that the flipped classrom based on rain classrom has better teaching effectiveness than routine flipped classrom. The questionnaire shows that the flipped classrom based on rain classrom will have more widely popularization in students and teachers.
\end{abstract}

\section{基于雨课堂的翻转教学模式研究}

张强 $1, \mathrm{a}$, 张雯 $2, \mathrm{~b},{ }^{*}$, 徐博 $2, \mathrm{c}$

1哈尔滨工程大学水下机器人技术重点实验室, 哈尔滨, 黑龙江, 中国

2哈尔滨工程大学自动化学院, 哈尔滨, 黑龙江, 中国

azhangqiang103@hrbeu.edu.cn, bzhangwen@hrbeu.edu.cn, ‘xubo@hrbeu.edu.cn

通讯作者

关键词：雨课堂；翻转课堂；教学体系；教学方案；考核方法

中文摘要. 针对翻转课堂推广难的问题, 以雨课堂为工具, 提出了基于雨课堂的翻转教学模 式, 建立了教、考一体化的教学体系, 研究了科学、客观的雨课堂翻转教学方案设计方法和 考核方法。教学实验表明雨课堂翻转教学模式的教学效果明显优于常规翻转课堂教学模式; 调查问卷表明基于雨课堂的翻转教学模式在教师和学生中具有较强的推广能力。

\section{1. 翻转课堂面临的问题}

翻转课堂颠覆了教师为课堂主体的传统教学模式, 采用课前学生利用视频、资料学习新 知识, 课堂上教师带领学生交流、讨论、做项目或实验的教学模式, 这种教学模式为学生提 供了更多接受指导的时间, 能够解决传统教学模式重理论轻实践、重知识传授轻能力培养的 问题 ${ }^{11,2]}$ 。随着新时期教学改革的不断深入, 近几年翻转课堂在网络上铺天盖地的传播, 但在 实际应用中翻转课堂却遭遇了推广难的尴矩 ${ }^{[3,4]}$, 其主要原因为: 制作微视频需要教师具有较 高的信息技术水平、教师课前准备耗时多、学生的自主学习任务完成率不高。 
雨课堂是清华大学和学堂在线共同推出的新型辅助教学工具, 它依托于广泛使用的微信 [5,6]。利用雨课堂提供的辅助教学功能, 教师可以通过电脑或手机将带有MOOC视频、PPT、 微信语音、视频的材料推送到学生手机, 使信息技术水平不足的教师能轻而易举的制作课前 教学材料, 同时缩短教师的课前准备时间; 另一方面, 利用雨课堂学生可通过手机随时进行 自主学习, 增强了学习的趣味性, 提高了学生的学习积极性。雨课堂具有使用便捷、推广能 力强的特点, 能够从教师和学生双方面解决在翻转课堂实施过程中出现的问题 ${ }^{[7]}$, 因此将雨 课堂应用于翻转课堂, 研究基于雨课堂的翻转课堂教学模式对全面推广翻转课堂有重要的意 义。

\section{2. 基于雨课堂的翻转教学模式}

考虑到只有教学方案和考核方法适配才能有效的衡量教学效果 ${ }^{[8]}$, 我们首先建立教、考 一体化的雨课堂翻转教学体系, 然后分别讨论相应的教学方案设计方法和考核方法。

\section{1 教、考一体化的雨课堂翻转教学体系}

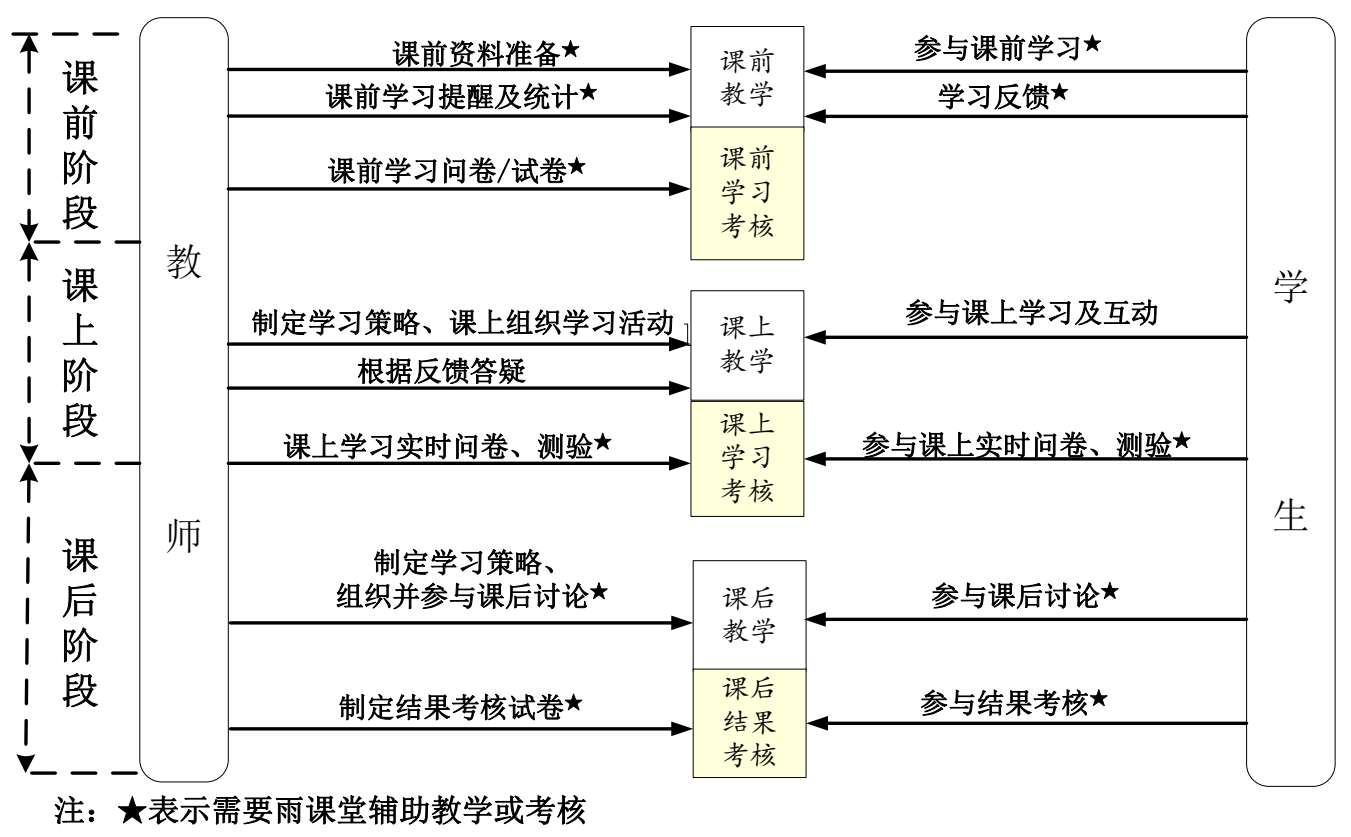

图1 教、考一体化的雨课堂翻转教学体系

根据雨课堂教学工具和翻转教学模式的特点, 采用由从整体到局部的原则, 建立教、考 一体化的雨课堂翻转教学体系, 如图1。该教学体系面向学生和教师, 一方面以学生为主体, 利用新颖的雨课堂工具提高学生的学习兴趣; 另一方面充分利用雨课堂的辅助教学功能, 最 大限度的为教师提供便捷，缩减教师的工作负荷。

如图1, 教、考一体化的雨课堂翻转教学体系分为课前教学、课上教学、课后教学三个阶 段, 其中课前以理论教学为主, 课上以知识内化和实践应用为主, 而课后讨论则作为前期学 习内容的延拓。考虑到课后结果考核不能体现学生的平时学习情况 ${ }^{[9]}$, 雨课堂翻转教学体系 采用全过程考核机制。在全过程考核机制中，学生的学习成绩是课前、课上学习过程考核和 课后结果考核的综合结果，其中过程考核由雨课堂工具实现即在课前和课上阶段教师利用雨 课堂的课前预习情况统计、到课率统计、课堂试题和问卷统计、问题讨论参与度统计等功能 进行学习过程考核。与传统的考核方式相比，基于雨课堂的过程考核不但是一种基于数据的 客观统计而且还可以为教师提供实时反馈, 方便教师及时调整教学方案、改善教学效果。 


\section{2 基于雨课堂的翻转教学方案设计}

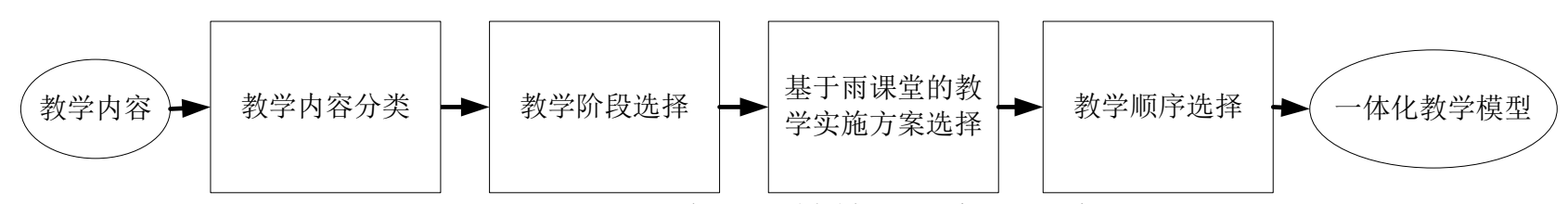

图2 基于雨课堂的翻转教学方案设计流程

以图1所示的教学体系为蓝本，以实现各教学阶段的有机结合为目标，按照如图2所示的 设计流程制定基于雨课堂的翻转教学方案：首先根据教学内容的分类方法对一节课所有的教 学内容进行分类。然后依照教学内容与教学阶段的适配关系为每项教学内容选择适当的教学 阶段。之后利用学习效果和教师工作负荷的综合评价为每项学习内容选择最适合的雨课堂辅 助教学工具。最后根据学生学习兴趣随学习时间的增加而降低的特点, 设计最优的教学顺序 使学生在整个教学过程中都维持浓厚兴趣 ${ }^{10]}$ 。

\section{3 基于雨课堂的翻转教学考核方法}

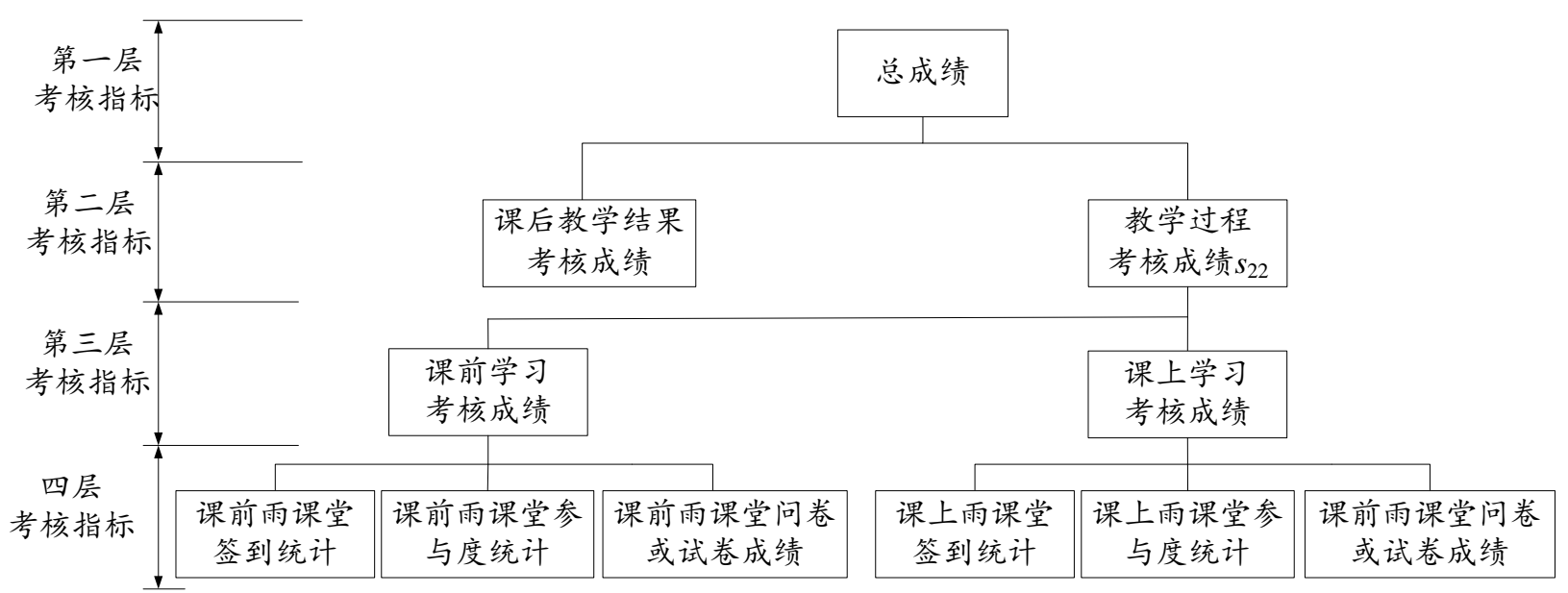

图3 基于雨课堂的翻转教学考核指标体系

翻转课堂的过程考核是一个多指标综合评估过程，可基于多指标综合评估理论，采用层 次分析法实现基于雨课堂的翻转教学全过程考核。具体方法为: 首先在雨课堂提供的统计指 标中挑选过程考核评价指标，形成如图3所示的考核指标体系。图3中考核总成绩包括课后教 学结果考核成绩和教学过程考核成绩两部分, 其中课后教学结果考核成绩即试卷考试成绩, 教学过程考核成绩由课前学习考核成绩和课上学习考核成绩组成, 课前和课上学习考核成绩 可由相应的雨课堂签到统计、参与度统计和问卷统计获得。根据层次分析法原理, 考核总成 绩可由图3中各层指标加权得到, 其中各指标的权值可利用专家打分的方法获得, 指标加权和 权值获取的具体方法参见文献[11]。

\section{3. 实验验证与调研}

为验证本文提出教学模式的实际应用效果, 以现代控制理论课程为例设计了基于雨课堂 的翻转教学方案, 并将其与常规翻转教学模式行进了对比实验。对比验证实验将参加实验的 75名学生随机、平均的分配成实验组和对照组。试验结果为: 实验组的课后考核平均成绩高 出对照组 8.9 分; 实验组的出勤率明显高于对照组, 实验组学生的课上参与度是对照组的 1.8 倍。其原因为雨课堂的PPT推送、弹幕、实时问答等功能激发了实验组学生的兴趣，而且雨 课堂的弹幕功能为实验组学生提供了随时参与课堂的机会。

为了验证雨课堂翻转教学模式的推广能力, 在 236 名学生和 45 名教师中间进行了该教学模 式的兴趣度问卷调查, 其中 $96 \%$ 的受访学生表示对雨课堂工具非常感兴趣, 能够利用雨课堂 
工具自主完成翻转课堂的课前和课后学习内容。 $85 \%$ 的受访教师认为雨课堂工具可缩小翻转 课堂课前准备的工作量和工作难度, 并表示愿意采用基于雨课堂的翻转教学模式。

\section{4. 总结}

针对翻转课堂教学模式在推广过程遭遇的问题, 构建了教、考一体化的雨课堂翻转教学 体系, 研究了雨课堂翻转教学方案设计的一般方法, 制定了基于雨课堂的翻转教学考核方法, 教学实验证明雨课堂翻转教学模式的教学效果优于传统翻转课堂教学模式, 调研表明基于雨 课堂的翻转教学模式继承了微信的广泛使用性, 是一种学生感兴趣、教师愿意采纳的教学模 式，因此具有广泛的推广潜力。

\section{致谢}

本文为哈尔滨工程大学教学改革研究项目《基于雨课堂的翻转课堂教学模式研究及实践》 (JG2017B07Z)和黑龙江省教育科学规划青年专项课题《面向全员的研究生国际化教育模式研 究与实践》（GBD1317119）的阶段性成果之一。

\section{References}

[1] Juhua Dou, Wen Shan, The teaching reform exploration of college english flipped classroom based on APP, Heilongjiang Researches on Higher Education, vol.5, pp. 162-167, 2015.

[2] Yin Da, Re-thinking on 'Flipped Classroom', China Information Technology Education, vol. 2, pp. 16, 2014.

[3] Anbao Xiao, Xie Jian, Fuqiang Gong, The application of rain classroom to flipped teaching in university course of ideological and political, Modern Educational Technology, vol. 5, pp. 46-52, 2017.

[4] Honge $\mathrm{Xu}$, Reflections on the promotion of localization teaching practice of 'Flipped Classroom', Journal of Hubei Normal University(Philosophy and Social Sciences), vol. 36, pp. 139-142, 2016.

[5] Shengxia $\mathrm{Xu}$, The teaching method keeps pace with times: Rain Classroom, Education Modernization, vol. 35, pp. 191-192, 2016.

[6] Shuaiguo Wang, Rain classroom: the wisdom teaching tool in the context of mobile internet and big data, Modern Educational Technology, vol. 5, pp. 26-32, 2017.

[7] Yao Jie, Weili Wang, Empirical study of the WeChat and Rain Classroom blended learning model applied in college teaching, Higher Education Exploration, vol. 9, pp. 50-54, 2017.

[8] Yangang Li, Huili Ma, Haijin Zhao, Teaching reform and practice of integrated teaching model-taking the course of "chemical Analysis" as an example, Chinese Journal of Chemical Education, vol.38, pp. 51-53, 2017.

[9] Xiaohong $\mathrm{Hu}$, Innovation of teaching mode based on process assessment-taking the course of " Ideological morality and legal foundation " as an Example, Studies in Ideological Education, vol. 4, pp. 50-54, 2013.

[10]Liyuan Shi, Psychological causes and regulation strategies of students' classroom silence, Teaching and Administration, vol. 22, pp. 29-31, 2017.

[11]Bingjiang Zhang, Analytic hierarchy process and application cases, Beijing: Publishing House of Electronics Industry, 2014. 